\title{
Effect of Different Beverages on the Physical Properties of Sonicfill Dental Composite
}

\author{
Lee KV로 , Philip $A^{1}$, Yahya NA² \\ ${ }^{1}$ Faculty of Dentistry, University of Malaya, Kuala Lumpur, Malaysia. \\ ${ }^{2}$ Department of Restorative Dentistry, Faculty of Dentistry, University of Malaya, Kuala Lumpur, Malaysia.
}

\begin{abstract}
Objective: To evaluate the effect of several beverages on the physical properties of SonicFill (Kerr Corp., USA), a new bulk-fill dental composite, over a period of time. Methods: A total of 28 discs (10 $\mathrm{mm} \times 2$ $\mathrm{mm})$ were prepared and randomly assigned into 4 groups $(n=7)$ according to the beverages they would be immersed in. The beverages chosen were Coca-Cola ${ }^{\odot}$, Nescafe ${ }^{\odot}$ coffee, Lipton tea $^{\odot}$ and distilled water (control). Surface roughness, microhardness and colour stability were evaluated using 3D optical surface texture analyser, Vickers microhardness tester and spectrophotometer respectively. Readings were recorded at the time intervals of 24 hours, 1 week and 1 moth after immersion. The data obtained were analysed using one-way ANOVA, repeated measures ANOVA and MANOVA. Results: There were significant differences in surface roughness for only two pairs of groups (distilled water and Coca-Cola, distilled water and coffee). However, there was no significant difference between the groups within the chosen time. Statistical analysis showed significant difference in microhardness between time only for Coca-Cola, with significant differences between 24 hours and 1 week; and 24 hours and 1 month. For the colour evaluation, there was significant difference between the groups within time. Conclusions: All the beverages chosen were able to affect the physical properties of the SonicFill. However, no particular beverage had a higher or lower impact on the surface roughness than the other beverages. Microhardness was affected by distilled water and Coca-Cola, in ascending order. Colour was affected most by coffee, followed by tea and Coca-Cola.
\end{abstract}

Keywords: Beverages, bulk-fill composite, colour stability, microhardness, surface roughness.

\section{INTRODUCTION}

Dental composite resins have been widely used in dentistry for over past many years due to the adhesion to the tooth structure, improvements in the mechanical properties and the diversity in colour options. In a long term clinical trial, it was found that the durability of these materials are satisfactory and their performance as posterior restorations is acceptable (1). However, composite restorations located in molars and posterior proximal have had a higher failure rate than other restoration sites. Dental composite resins have been frequently altered in their composition in the past years due to technological advancements. Currently, clinicians have an increasing preference for posterior composite restorations that involve simplify procedures and shorten clinical chairside time.

Recently, bulk-fill composites - a new category of resin composites -, have been introduced in the dental market. The restoration process is sped up 
by ultra-efficient curing enabling thicknesses up to $4 \mathrm{~mm}$ increments to be cured in a single step, thereby reducing chairside time via eliminating the amount of layering steps, compared to conventional composites which require placement and curing of 2 $\mathrm{mm}$ increments (2).

A comparison study has been conducted to evaluate the physico-mechanical properties of the presently available bulk-fill composites with commercially available nano-hybrid composites (both in paste and flowable forms). The study concluded that bulk-fill composite had the superiority in time-saving and easy handling; however it has limitations in the mechanical properties when compared to other nano-hybrid composites (3). In another comparison study between different nano-hybrid bulk-fill composites, SonicFill ${ }^{\mathrm{TM}}$ scored the highest among the tested materials in terms of mechanical properties and can be used as an alternative to conventional composite for restorations of posterior teeth (4). There are correlations between the thickness of resin and the microhardness and optical properties of bulk-fill composites where these properties decreased as the resin thickness increased (5).

As the resin restorative materials were placed in the oral environment, they were exposed to a great number of adverse conditions, challenging their integrity and durability over time. Consumption of certain beverages, such as soft drinks, tea, coffee, and alcohol, may influence the aesthetic and the physical properties of composites, thereby undermining the quality of the restorations (6-9). Beverages may have more of an effect depending on the intrinsic features of the restorative materials, such as the chemical composition (10). Furthermore, the effect of beverages on the mechanical properties of the composites may be complementary to the amount and frequency of its intake. The chemicals in common beverages can have a significant influence on surface degradation and lead to wear of composite restorations, resulting in compromised aesthetics due to external pigmentations on resin restoration, decreasing their hardness, and facilitating the wear of their surfaces with the course of time $(6,7,11)$. A reduction in the microhardness value may signify a superficial degradation, and therefore a change in its roughness, which correlates with the plaque accumulation and followed by deposition of lactic acid, thus jeopardizing the restoration durability (12, $13,14,15)$.

Nowadays bulk fill composites have become widely used among the clinicians. However, only few studies have been published on the effect of beverages on the aesthetics and physical properties of the bulk-fill composites. Therefore, the aim of this study was to evaluate the effect of several beverages on the physical properties (surface roughness, microhardness, and colour stability) of a new bulk-fill composite resin [SonicFill ${ }^{\mathrm{TM}}$ (Kerr Corp., USA)] over a period of 1 month. The null hypothesis of this study was that there is no difference in surface roughness, microhardness, and colour stability for SonicFill when immersed in different beverages.

\section{MATERIALS AND METHODS}

\section{Sample preparation}

The nano-hybrid, bulk-fill composite, SonicFill ${ }^{\mathrm{TM}}$ (Kerr, USA) (Table 1), was used in the preparation of the specimens. The composite was packed into a customised stainless steel mould with $10 \mathrm{~mm} \times 2$ $\mathrm{mm}$ holes. The top surface was covered with a Mylar strip followed by a glass slide (1 $\mathrm{mm}$ in thickness). Constant finger pressure was applied on the slide to accommodate the material into the mould, extrude excess material and ensure the samples had a flat surface. After removing the slide, each specimen was light-cured from the top surface for 40 seconds using Demi PlusLED light curing unit (Kerr, USA) under the standard curing mode output i.e. 450$470 \mathrm{~nm}$ wavelength range and $1330 \mathrm{~mW} / \mathrm{cm}^{2}$ output irradiance. The specimens were then removed from the mould and the bottom surfaces were light cured. Each specimen was finished and polished in a standardized manner, using a series of Sof-Lex ${ }^{\mathrm{TM}}$ (3M ESPE, St. Paul, MN, USA) polishing discs, starting from the coarsest to the finest discs. The debris were removed from the specimens' surfaces using an ultrasonic bath cleaner and blotted dried with filter papers.

A total of 28 disc-shaped specimens were fabricated and 4 groups $(n=7)$ were formed. Each group of specimen was then placed in a standard plastic tube and stored in an incubator set at $37^{\circ} \mathrm{C}$ for 24 hours prior to the testing for baseline readings. After the baseline readings were recorded, each group of specimen were then immersed in the immersion media (Table 2) and returned to the incubator. The media used were replaced every 3 days to avoid microbial activity and the tubes were rotated daily to prevent stagnation of the media. The surface roughness, microhardness and colour stability readings were taken at baseline (before immersion) and at 24 hours (day 1), 1 week (day 7 ), and 1 month (day 28) after initial immersion. Prior to taking readings, the specimens' discs were 
washed in distilled water and blotted dry. After the tests, the specimens were returned to their tubes and immersed in the respective media.

The preparation of tea and coffee were standardized. The coffee solution was prepared with one teaspoon of granulated coffee in $180 \mathrm{ml}$ of boiled distilled water (manufacturer recommendation). Meanwhile the tea solution was prepared by immersing a Lipton tea bag in $200 \mathrm{ml}$ of boiled distilled water for 2 minutes, dipping the tea bag in and out 10 times, and then removing it. Special precautions

Table 1: Bulk-fill composite used in this study.

\begin{tabular}{lllllll}
\hline Brand & Manufacturer & $\begin{array}{l}\text { Material } \\
\text { category }\end{array}$ & $\begin{array}{l}\text { Resin } \\
\text { matrix }\end{array}$ & $\begin{array}{l}\text { Filler } \\
\text { composition }\end{array}$ & $\begin{array}{l}\text { Filler volume } \\
(\%)(w t)\end{array}$ & Shade \\
\hline SonicFill ${ }^{\text {TM }}$ & $\begin{array}{l}\text { Kerr Corp., } \\
\text { Orange, CA, }\end{array}$ & Nano-hybrid & Bis-GMA, & $\mathrm{SiO}_{2}$, glass, & $83.5 \%$ & $\mathrm{~A} 2$ \\
& & TEGDMA & oxide & & \\
\hline
\end{tabular}

Table 2: Immersion media used in the present study.

\begin{tabular}{llll}
\hline Material & Brand & Manufacturer & Principle Ingredients \\
\hline $\begin{array}{l}\text { Distilled water } \\
\text { (control) }\end{array}$ & - & Laboratory & Water \\
Cola soft drink & Coca-Cola $^{\odot}$ & $\begin{array}{l}\text { F\&N Beverages, Manufacturing Sdn. Bhd., } \\
\text { Shah Alam, Selangor, Malaysia }\end{array}$ & $\begin{array}{l}\text { Carbonated water, } \\
\text { sugar, caramel, cola } \\
\text { nut extract, caffeine, } \\
\text { colouring. }\end{array}$ \\
Coffee & Nescafe $^{\odot}$ classic & $\begin{array}{l}\text { Nestle products Sdn. Bhd., Petaling Jaya, } \\
\text { Selangor, Malaysia }\end{array}$ & Coffee beans \\
Tea & Lipton $^{\circledR}$ yellow \\
label tea bags & $\begin{array}{l}\text { Unilever Foods (Malaysia) Sdn. Bhd., } \\
\text { Kuala Lumpur, Malaysia }\end{array}$ & $100 \%$ Tea Leaves \\
\hline
\end{tabular}

were taken when dispensing the Coca-Cola in order to maintain the carbonic gas. All the media were utilized at room temperature, approximately $28^{\circ} \mathrm{C}$.

\section{Surface roughness evaluation}

The reading of the surface roughness $\left(R_{a}\right)$ was obtained using the $3 \mathrm{D}$ Optical Surface Texture Analyzer (ALICONA, InfiniteFocus Real3D, Belgium). Readings were conducted at 100x magnification in five different locations on each sample. At least five readings were taken from each location. An average was then taken giving five values for each sample, and then a further average to whittle the result to one value of $R_{a}$ (in $\mu \mathrm{m}$, accurate to three decimal points) per sample.

\section{Vickers microhardness evaluation}

The Vickers microhardness number (VHN) was obtained using a diamond penetrator in the shape of a pyramid with a square base and an angle of $136^{\circ}$, applying a $100 \mathrm{~g}$ load for 10 seconds. The procedure was performed in five different random places on the upper surface of each specimen using a microhardness tester (Shimadzu Corp., Kyoto, Japan.). Five VHNs (accurate to one decimal point) were obtained from each sample, which were then pared down into one average value.

\section{Colour stability evaluation}

The colour evaluation was done using spectrophotometer (Konica Minolta Spectrophotometer CM-5, Germany), where each sample was placed on the illumination area (a circle of $3 \mathrm{~mm}$ diameter) and the reflectance values were measured after 1 second of being illuminated by a pulse xenon lamp. There was one reading per sample. The measurements obtained were $L^{*}, a^{*}$, $b^{*}$; respectively they represent the lightness of the colour $\left(\mathrm{L}^{*}=0\right.$ yields black and $\mathrm{L}^{*}=100$ indicates diffuse white; specular white may be higher), its position between red/magenta and green $\left(a^{*}\right.$, negative values indicate green while positive values 
indicate magenta) and its position between yellow and blue ( $b^{*}$, negative values indicate blue and positive values indicate yellow). The reading used for the purposes of this study was the $L^{*}$ reading (accurate to two decimal points), which is similar to the value parameter of the Munsell colour model.

\section{Statistical analysis}

The average of the surface roughness, the average of the microhardness, and the colour change at 24 hours, 1 week, and 1 month were calculated for each group of specimens (with their different immersion solutions). After the normality assumptions were tested and met, analysis was carried out using IBM SPSS Statistics software $v 22.0$ for Windows with the one-way ANOVA, repeated measures ANOVA and multivariate analysis of variance (MANOVA) models.

\section{RESULTS}

Figure 1 illustrates a line graph of surface roughness of SonicFill ${ }^{\mathrm{TM}}$ immersed in different immersion media over a period of time. It can be seen from the graph that at 24 hours and 7 days, the surface roughness values for all groups were approximately in the range of 0.1 to $0.2 \mu \mathrm{m}$. However, at 1 month, the surface roughness for the composite immersed in the distilled water increased to $0.24 \pm 0.11 \mu \mathrm{m}$.

\section{Surface Roughness:}

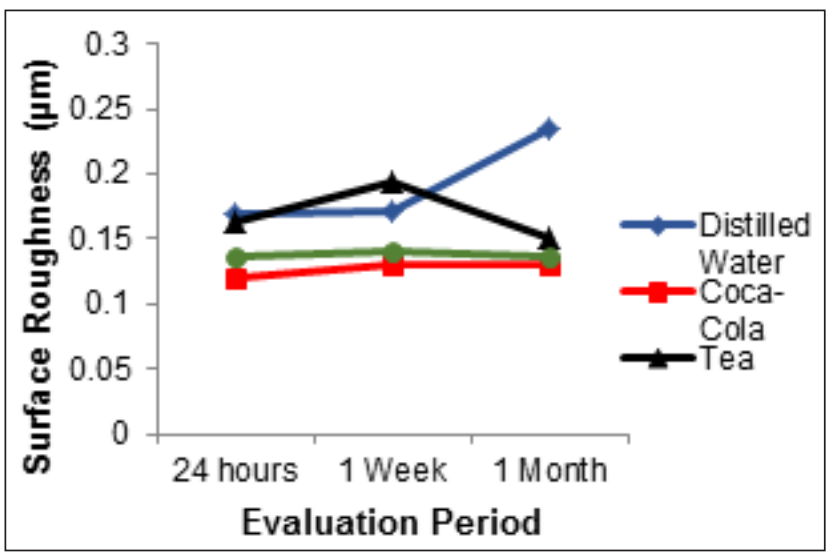

Figure 1: Line graph showing change of surface roughness $(\mu \mathrm{m})$ over time.

Overall, there was a significant difference $(p=0.002)$ of surface roughness between groups (distilled water, Coca-Cola, tea, coffee). Further analysis using Bonferroni correction showed that there were significant differences for only two pairs of groups (distilled water and Coca-Cola, $p=0.002$; distilled water and coffee, $p=0.024)$. However, there was no significant difference between the groups within time $(p=0.311)$.

\section{Microhardness}

Figure 2 shows the mean value of microhardness in VHN of the specimens over a period of time. All the 3 tested groups (Coca-Cola, tea and coffee), consistently showed lower VHN compared to the control group (distilled water) throughout the evaluation period. The group immersed in tea showed the lowest VHN overall. Regarding the microhardness of the samples, statistical analysis showed significant difference between time only for Coca-Cola $(p=0.008)$, with significant differences between 24 hours and 1 week $(p=0.024)$ and 24 hours and 1 month $(p=0.022)$. There was significant difference between groups $(p=0.002)$, and further analysis with Bonferroni correction showed that three pairs of groups had significant differences (distilled water and Coca-Cola, $p=0.029$, distilled water and tea, $p=0.003$, distilled water and coffee, $p=0.022$ ). There was no significant difference between the groups within time $(p=0.849)$.

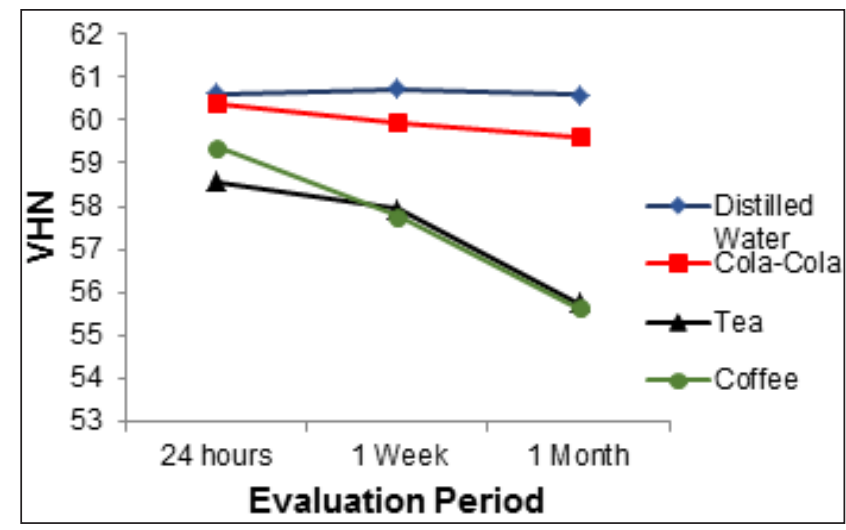

Figure 2: Line graph showing change of microhardness (VHN) over time.

\section{Colour stability}

The colour stability of SonicFill ${ }^{\mathrm{TM}}$ over a period of time is shown in Figure 3. Both tea and coffee groups, showed a marked decreased in colour stability over a period of time. However, for samples immersed in both distilled water and Coca-Cola, the colour changes were very minimal.

For the colour evaluation, it was found that there was significant difference between time for Coca-Cola between 24 hours and 1 week $(p=0.002)$ 


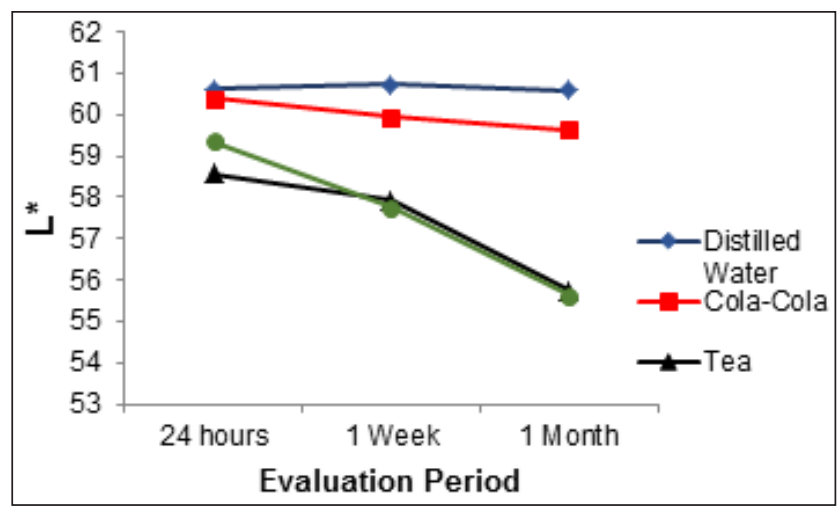

Figure 3: Comparative mean colour stability values for distilled water, Coca-Cola, tea, and coffee.

and between 24 hours and 1 month $(p=0.000)$; for tea between 24 hours and 1 week $(p=0.004)$ and between 24 hours and 1 month $(p=0.000)$; for coffee between 24 hours and 1 week $(p=0.039)$ and between 24 hours and 1 month $(p=0.000)$. There was an overall significant difference between groups $(p=0.000)$. Further analysis using Bonferroni correction showed significant differences between four pairs of groups (distilled water and Coca-Cola, $p$ $=0.023$, distilled water and coffee, $p=0.063$, CocaCola and tea, $p=0.000$, Coca-Cola and coffee, $p=$ $0.000)$.

There was significant difference between the groups within time $(p=0.000)$. Within 24 hours, the following group pairs had significant means between them; distilled water and Coca-Cola, distilled water and tea, distilled water and coffee, Coca-Cola and tea. Within 1 week, the following group pairs had significant means between them; distilled water and Coca-Cola, distilled water and coffee, Coca-Cola and tea, Coca-Cola and coffee, tea and coffee. Within 1 month, the following group pairs had significant means between them; distilled water and Coca-Cola, distilled water and tea, distilled water and coffee, Coca-Cola and tea, Coca-Cola and coffee.

\section{DISCUSSION}

The nature of the resin composites is dependent on the composition and filler distribution in the matrixes. Factors that influence the characteristics of composites include resin monomers, fillers, and coupling agents. There is direct correlation between filler content and the depth of polymerization, hardness, compressive strength, colour stability, as well as stiffness. Increased filler loading has been shown to result in less surface degradation due to lower water adsorption (16).

In this study, a newly introduced bulk-fill composite, SonicFill ${ }^{\mathrm{TM}}$ (Kerr Corp., USA) (Figure 4) has been used. The SonicFill ${ }^{T M}$ system consists of a handpiece that dispenses the composite. The handpiece, delivers sonic energy at varying intensities, which is adjusted on the shank from low to high ( 1 to 5 ) to control rate of composite extrusion. The composite incorporates modifiers that react to sonic vibrations to alter the viscosity of the material. The single dose capsules have smaller diameter with $1.5 \mathrm{~mm}$ tips for accessing deep cavities. The resin dispensing tips screw directly onto the handpiece head and deliver the composite when activated by the foot control. The sonic energy reduces the viscosity of the resin by $87 \%$ allowing adaptation in deep cavities, up to $5 \mathrm{~mm}$, in a single increment. After the foot control is released the sonic energy ceases, and the resin returns to its high viscosity state, facilitating sculpting and carving to the desired anatomical form.

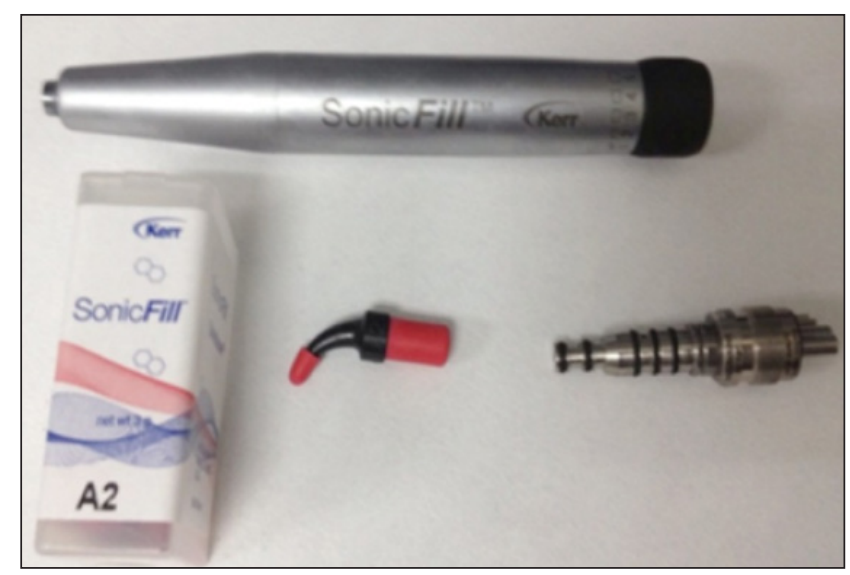

Figure 4: SonicFillTM bulk-fill composite system.

This study sought to investigate the effect of different beverages - namely, Coca-Cola, tea, and coffee - on the surface roughness, microhardness, and colour stability of the SonicFill ${ }^{\mathrm{TM}}$ bulk fill composite within the time period of 24 hours, 1 week, and 1 month. In this study, it was recorded that the roughness values for Coca-Cola, coffee, tea and distilled water were significant. Coca-cola has the erosive potential with $\mathrm{pH}$ that is low 2.5 (17). This soft drink contains a strong inorganic acid, phosphoric acid $\mathrm{H}_{3} \mathrm{PO}_{4}$. The existence of a low $\mathrm{pH}$ value must have resulted in a more aggressive attack on the surface of the restorative material, 
leading to a greater surface roughness. Meanwhile, the significant roughness value for coffee could theoretically be due the fact that the coffee used in our study was unfiltered and instant; although there is no controlled study to support this suggestion, it can be speculated that the absorption of the size and distribution of organic particles of coffee may have led to alterations in the surface roughness of the composite. There was, however no significant increase in surface roughness of SonicFill ${ }^{\mathrm{TM}}$ between 24 hour, 1 week, and 1 month. This could be due to the higher filler loading of SonicFill ${ }^{\mathrm{TM}}$ which increases the stability of the resin composite against low $\mathrm{pH}$ conditions $(18,19)$. Unexpected increased in surface roughness value in the control group after 1 month period is difficult to comprehend. Repeating this part of the experiment therefore is needed to reconfirm the current findings.

Hardness is defined as the resistance of the material to indentation (20). Water sorption and hydrolytic degradation can influence the microhardness surface of any resin composite (21). As additional information, the microhardness of the SonicFill measured after 24 hours was higher than the baseline hardness value. This could be due to the increased conversion of monomers and/or postcuring cross-linking reactions in the resin phase over that period of time $(22,23,24)$.

Regarding the results obtained, it was found that all the beverages had significant differences to the results of the distilled water group. This does coincide with the expectation that the microhardness should not (and did not) decrease significantly, while the microhardness of the Coca-Cola, tea, and coffee groups decreased significantly in comparison. Of the three, though, only the readings of the CocaCola group showed a significant decrease in microhardness values after 1 week and 1 month. This could be due to the erosion potential of the acids in the soft drink which penetrate the resin matrix, resulting in the release of any unreacted monomers and thereby lowering the microhardness value $(25$, 26).

In this study, colour change was measured via lightness change $L^{*}$; simply a quantitative measure of whether the samples became darker or lighter as the study went on. The values of the SonicFill ${ }^{\mathrm{TM}}$ samples immersed in Coca-Cola, tea, and coffee showed a significant difference from the beginning to the end of the study. Coffee demonstrated the greatest colour change followed by Coca-Cola - as shown by the significant differences between those groups and the control group. Extrinsic factors for colour change include the staining effect of colorants by adsorption or absorption from exogenous sources (27). These extrinsic factors cause staining of the restorative materials and oral tissues, notably in combination with dietary factors; among these factors, coffee, tea, and nicotine have been reported $(27,28)$. The greater colour change in coffee could be due to the adsorption on as well as absorption of the yellow colorant in coffee onto the resin composite materials. The discolouration due to tea was induced only by adsorption of colorant onto the resin composite materials. Coca-Cola had the lowest colour change compared to coffee and tea due to its lack of yellow colorant (27).

Within 1 month, all the groups had a significant difference in means between them and the control group, as should be expected. Additionally, there were significant differences in mean between CocaCola and tea as well as Coca-Cola and coffee, suggesting that the degree of colour change of the Coca-Cola group was not as significant as that of the tea and coffee groups. As an aside, it was observed that the unpolished surfaces of the samples had a qualitatively greater colour change, possibly correlating colour change to surface roughness, though there is no independent study to support this hypothesis.

\section{CONCLUSION}

Based on the results obtained from this study, it can be concluded that beverages have a significant effect on the surface roughness, microhardness, and colour of the nano-hybrid, bulk fill composite SonicFill. No particular beverage had a higher or lower impact on the surface roughness than the other beverages. Microhardness was affected by distilled water and Coca-Cola, in ascending order. Colour was affected most by coffee, followed by tea and Coca-Cola.

Further laboratory testing to determine the effects of these same beverages on the characteristics of other composite resins should be done to compare the efficacy of SonicFill bulk fill composite with its competitors on the market.

\section{ACKNOWLEDGEMENTS}

The authors would like to thank Miss Najihah Lokman for her kind help with the SPSS software and the statistical analysis. We also would like to thank Miss Norazira Othman and all staf of Biomaterials Research Laboratory for their assistance in the 
running of the experiment. This research is supported by University of Malaya Research Grant RG 53113HTM.

\section{DECLARATION OF INTEREST}

The authors alone are responsible for the content of this article, and report no conflicts of interest.

\section{REFERENCES}

1. da Rosa Rodolpho PA, Cenci MS, Donassollo TA, Loguercio AD, Demarco FF. A clinical evaluation of posterior composite restorations: 17-year findings. J Den. 2006; 34(7): 427-35.

2. Czasch $P$, llie $N$. In vitro comparison of mechanical properties and degree of cure of bulk fill composites. Clin Oral Invest. 2013; 7(1): 227-35.

3. Leprince JG, Palin WM, Vanacker J, Sabbagh, J, Devaux J, Leloup G. Physico-mechanical characteristics of commercially available bulkfill composites. J Dent. 2014; 42(8): 993-1000.

4. Didem A, Gözde Y, Nurhan, Ö. Comparative Mechanical Properties of Bulk-Fill Resins. Open J Comp Mater. 2014; 4: 117-21.

5. Kim EH, Jung KH, Son SA, Hur B, Kwon $\mathrm{YH}$, Park JK. Effect of resin thickness on the microhardness and optical properties of bulk-fill resin composites. Rest Dent Endo. 2015; 40(2): 128-35.

6. Chan KC, Fuller JL, Hormati AA. The ability of foods to stain two composite resins. J Prosthet Dent. 1980; 43(5): 542-5.

7. Dietschi D, Campanile G, Holz, J, Meyer JM. Comparison of the color stability of ten newgeneration composites: an in vitro study. Dent Mater. 1994; 10(6): 353-62.

8. Lim BS, Moon HJ, Baek KW, Hahn SH, Kim CW. Color stability of glass-ionomers and polyacid-modified resin-based composites in various environmental solutions. Am J Dent. 2001; 14(4): 241-6.

9. Wiltshire WA, Labuschagne PW. Staining of light-cured aesthetic resin restorative materials by different staining media: an in vitro study. $\mathrm{J}$ Dent Assoc S Afr. 1990; 45(12): 561-5.

10. Ameye C, Lambrechts P, Vanherle G. Conventional and microfilled composite resin Part 1 Colour stability and marginal adaptation. J Prosthet Dent. 1981;46(6):623-30.
11. Powers JM, Fan PL. Erosion of composite resins. J Dent Res. 1980; 59(5): 815-19.

12. Badra VV, Faraoni JJ, Ramos RP, Palma-Dibb RG. Influence of different beverages on the microhardness and surface roughness of resin composites. Oper Dent. 2005; 30(2): 213-9.

13. Chinelatti MA, Chimello DT, Ramos RP, PalmaDibb RG. Avaliação da dureza superficial de resinas compostas antes e após o polimento em diferentes tempos. J Appl Oral Sci. 2006; 14(3): 188-92.

14. Diaz-ArnoldAM, Wistrom DW, SwiftEJ Jr. Topical fluoride and glass ionomer microhardness. Am J Dent. 1995; 8(3): 134-6.

15. Samuel SM, Rubinstein C. Microhardness of enamel restored with fluoride and non-fluoride releasing dental materials. Braz Dent J. 2001; 12(1): 35-8.

16. Kim KH, Ong JL, Okuno O. The effect of filler loading and morphology on the mechanical properties of contemporary composites. J Prosthet Dent. 2002; 87(6): 642-9.

17. Al-Shalan, TA. In vitrostaining of nanocomposites exposed to a cola beverage. Pakistan Oral Dent J. 2009; 29(1).

18. Soderholm KJ. Leaking of fillers in dental composites. J Dent Res. 1983; 62(2): 126-30.

19. Han L, Okamoto A, Fukushima M, Okiji T. Evaluation of flowable resin composite surfaces eroded by acidic and alcoholic drinks. Dent Mater J. 2008; 27(3): 455-65.

20. Yap AU, Mah MK, Lye CP, Loh PL. Influence of dietary simulating solvents on the hardness of provisional restorative materials. Dent Mater. 2004; 20(4): 370-6.

21. Chadwick RG, McCabe JF, Walls AWG, Storer $R$. The Effect of Storage Media upon the Surface Microhardness and Abrasion Resistance of Three Composites. Dent Mater, 1990; 6: 123-8.

22. Milleding $P$, Ahlgren $F$, Wennerberg $A$, Ortengren, U, Karlsson, S. Microhardness and surface topography of a composite resin cement after water storage. Int J Prosthodont. 1998; 11(1): 21-6.

23. De Moraes RR, Marimon, JLM, Jochims Schneider LF, Sinhoreti, MAC, Correr-Sobrinho L, Bueno M. Effects of 6 Months of Aging in Water on Hardness and Surface Roughness of Two Microhybrid Dental Composites. J Prosthodont. 2008; 17: 323-26.

24. Yanikoglu N, Duymus ZY, Yilmaz B. Effects of different solutions on the surface hardness of composite resin materials. Dent Mater J. 2009; 28(3): 344-51. 
25. Nicholson JW, Gjorgievska E, Bajraktarova B, McKe:zie MA. Changes in properties of polyacidmodified composite resins (compomers) following storage in acidic solutions. J Oral Rehabil. 2003; 30(6): 601-7.

26. Yesilyurt C, Yoldas O, Altintas $\mathrm{SH}$, Kusgoz A. Effects of food-simulating liquids on the mechanical properties of a silorane-based dental composite. Dent Mater J. 2009; 28(3): 362-7.

27. Um CM, Ruyter IE. Staining of resin-based veneering materials with coffee and tea. Quint Int. 1991; 22(5): 377-86.

28. Prayitno $S$, Addy M. An in vitro study of factors affecting the development of staining associated with the use of chlorhexidine. J Periodent Res. 1979; 14: 397-402.

\section{Corresponding author:}

\section{Dr Noor Azlin Yahya}

Department of Restorative Dentistry,

Faculty of Dentistry, University of Malaya, 50603, Kuala Lumpur, Malaysia.

Tel: +603-7967 7441

E-mail Address: nazlin@um.edu.my 